\title{
Generic Neutravidin Biosensor for Simultaneous Multiplex Detection of MicroRNAs via Electrochemically Encoded Responsive Nanolabels
}

Sawsen Azzouzi, ${ }^{\dagger, \ddagger, \S, \|}$ Zina Fredj, ${ }^{\dagger, \ddagger, \S, \|}$ Anthony P. F. Turner, ${ }^{\dagger, \perp}$ Mounir Ben Ali, ${ }^{\dagger, \S}$ and Wing Cheung Mak*, ${ }^{*}$

${ }^{\dagger}$ Biosensors and Bioelectronics Centre, Department of Physics, Chemistry and Biology (IFM), Linköping University, S-58183 Linköping, Sweden

${ }^{\ddagger}$ Higher Institute of Applied Sciences and Technology of Sousse, GREENS-ISSAT, University of Sousse, Cité Ettafala, Ibn Khaldoun 4003 Sousse, Tunisia

${ }^{\S}$ NANOMISENE Lab, LR16CRMN01, Centre for Research on Microelectronics and Nanotechnology of Sousse, Technopole of Sousse B.P. 334, Sahloul 4034, Sousse, Tunisia

Supporting Information

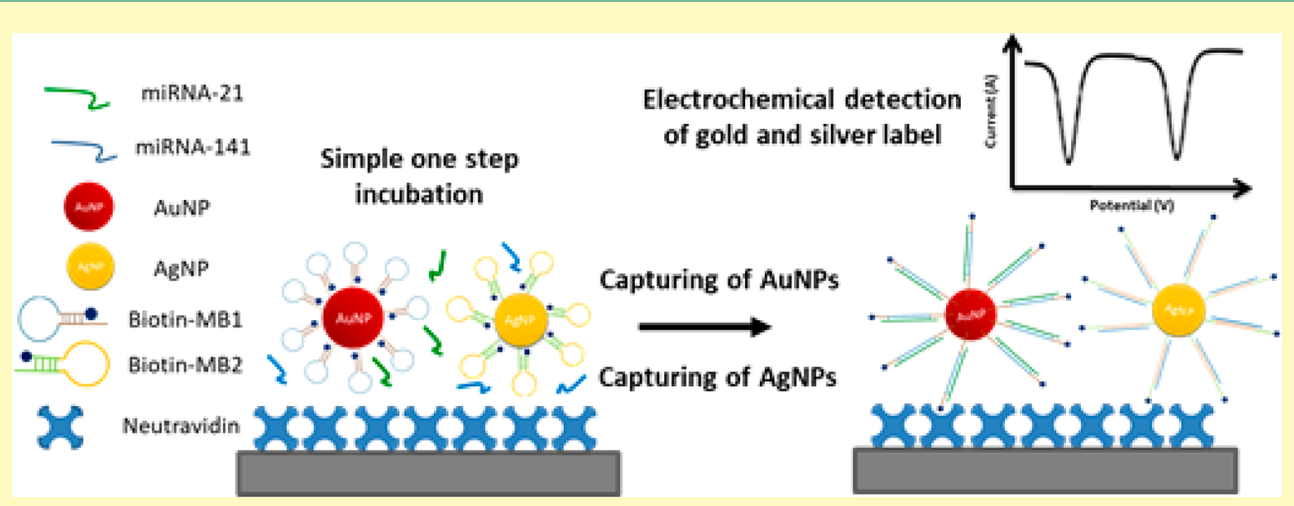

ABSTRACT: Current electrochemical biosensors for multiple miRNAs require tedious immobilization of various nucleic acid probes. Here, we demonstrate an innovative approach using a generic neutravidin biosensor combined with electrochemically encoded responsive nanolabels for facile and simultaneous multiplexed detection of miRNA-21 and miRNA-141. The selectivity of the biosensor arises from the intrinsic properties of the electrochemically encoded responsive nanolabels, comprising biotinylated molecular beacons (biotin-MB) and metal nanoparticles (metal-NPs). The procedure is a simple one-pot assay, where the targeted miRNA causes the opening of biotin-MB followed by capturing of the biotin-MB-metal-NPs by the neutravidin biosensor and simultaneous detection of the captured metal-NPs by stripping square-wave voltammetry (SSWV). The multiplexed detection of miRNA-21 and miRNA-141 is achieved by differentiation of the electrochemical signature (i.e., the peak current) for the different metal-NP labels. The biosensor delivers simultaneous detection of miRNAs with a linear range of $0.5-1000 \mathrm{pM}$ for miRNA-21 and a limit of detection of $0.3 \mathrm{pM}$ ( $3 \sigma /$ sensitivity, $n=3$ ), and a range of $50-1000 \mathrm{pM}$ for miRNA-141, with a limit of detection of $10 \mathrm{pM}$. Furthermore, we demonstrate multiplexed detection of miRNA-21 and miRNA-141 in a spiked serum sample.

KEYWORDS: neutravidin biosensor, multiplex detection, microRNAs, molecular beacons, nanoparticle labels

M icroRNAs (miRNAs) are small endogenous nonprotein coding RNAs (approximately 17-25 nucleotides) and were first discovered in 1993 by Lee et al. ${ }^{1}$ and Wightman et $\mathrm{al}^{2}$ from Caenorhabditis elegans as the lin-14 miRNA. A few years later, in 2000, Pasquinelli et al. reported the discovery of human miRNA as let-7 miRNA. ${ }^{3}$ miRNAs play commanding roles in several cellular processes such as differentiation, proliferation, metabolism, and apoptosis. ${ }^{4-6}$ Recent studies have correlated the alteration in expression levels of extracellular miRNAs in body fluids as emerging biomarkers for various cancers, ${ }^{7,8}$ as well as other human diseases such as cardiovascular diseases, ${ }^{9}$ autoimmune diseases, ${ }^{10}$ and neurodegenerative diseases. ${ }^{11}$ Moreover, extracellular miRNAs are highly stable against temperature, $\mathrm{pH}$, and storage, ${ }^{12}$ which makes extracellular miRNAs attractive biomarkers for diagnostics and prognostic applications. ${ }^{13,14}$

Various analytical techniques have been developed for sensitive quantification of miRNAs such as Northern blot, ${ }^{15}$

Received: August 31, 2018

Accepted: January 24, 2019

Published: February 7, 2019 
quantitative real time PCR (qRT-PCR), ${ }^{16}$ microarrays, ${ }^{17}$ and surface-enhanced Raman scattering (SERS). ${ }^{18}$ Although these methods can adequately monitor the levels of microRNAs, they require skilled personnel and sophisticated and expensive instrumentation. In contrast, electrochemical biosensors possess analytical and economic advantages including simple instrumentation, low-cost, high sensitivity, fast response, and easy operation. ${ }^{19}$ In this context, various electrochemical biosensors for detection of miRNAs have been developed. Bettazzi et al. reported an electrochemical method for miRNA222 detection based on paramagnetic beads and enzyme amplification. ${ }^{20}$ Salahandish et al. developed an ultrasensitive nanogenosensor for the detection of miRNA-21 based on sandwiched gold nanoparticles (AgNPs) in PANI and $\mathrm{N}$ doped graphene. ${ }^{21}$ Yin et al. demonstrated the electrochemical determination of miRNA-21 based on a graphene-modified glassy carbon electrode and LNA integrated molecular beaconAuNPs. ${ }^{22}$ Kangkamano et al. established a label-free electrochemical miRNA biosensor based on a pyrrolidinyl peptide nucleic acid and a silver nanofoam (AgNF) modified electrode for detection of miRNA/21. ${ }^{23}$ However, these reported electrochemical biosensors usually required tedious chemical procedures for the immobilization of the oligonucleotide probe onto electrode surface. Recently, we reported the use of a simple neutravidin biosensor exploiting a dual-functional biotin-molecular beacon-AuNP nanolabel for sensitive detection of microRNA- $21 .^{24}$

For the development of a multiplexed electrochemical biosensor for the detection of different miRNAs, the immobilization chemistry required becomes more complex, necessitating the coimmobilization of multiple complementary oligonucleotide probes for different individual miRNA targets. Feng et al. developed an electrochemiluminescence biosensor array with coimmobilized multiple capture probes for multiplexed detection of miRNA-141 and miRNA-21 using doped silica nanolabels. ${ }^{25}$ However, the so-called multiplex detection system was performed with two separated working electrodes. Ghazizadeh et al. reported the use of liposomes functionalized with multiple oligonucleotide capturing probes for the multiplexed electrochemical detection of miRNA-21, miRNA-124a and miRNA-221. ${ }^{26}$ However, this approach suffered from a similar drawback, since the multiplexed electrochemical detection was performed either with three individually separated working electrodes or sequentially target-by-target with multiple measuring steps. Zhu et al. developed a label-free electrochemical assay using multiple capture probes on functionalized magnetic beads coupled with quantum dots barcoded via the ligase chain reaction for multiplex detection of miRNA-155 and miRNA-27b, but the ligase chain reaction-treated magnetic beads needed to be incubated at a high temperature of $90{ }^{\circ} \mathrm{C} .{ }^{27}$ Yuan et al. demonstrated the simultaneous electrochemical detection of miRNA-141 and miRNA-27b on a single gold electrode with immobilized multiple capture probes coupled with magnetic nanolabels driven by additional hybridization chain reaction steps. $^{28}$ Yang et al. reported a multiplexed electrochemical biosensor for miRNA-141 and miRNA-21 fabricated by immobilizing multiple capturing probes onto a gold disc electrode coupled with different redox labels followed by signal generation via duplex specific nuclease-assisted amplifications. $^{29}$ However, most of the reported electrochemical techniques for multiplexed detection of miRNAs still require relatively tedious and complex procedures for fabrication of electrochemical transducers which includes coimmobilization of multiple oligonucleotide capture probes and demand additional hybridization or amplification steps. In addition, the immobilization of oligonucleotide probes is less efficient due to the intrinsically linear structure of the oligonucleotide; ${ }^{30}$ thus, the preparation of biosensors for the detection of multiple miRNAs or DNA targets with multiple oligonucleotide probes remains difficult.

We report herein the use of a simple generic neutravidin modified electrode combined with electrochemically encoded responsive nanolabels for simultaneous multiplexed detection of miRNA-21 and miRNA-141. The electrochemically encoded responsive nanolabels consisted of AuNPs and AgNPs coupled with biotin-MB. The immobilization chemistry and preparation of neutravidin modified electrodes is relatively simple, and more importantly, the multiplexing capacity of such simple neutravidin biosensor is not limited by the complicity encountered in the conventional coimmobilization multiple oligonucleotide probes approaches. The fabrication and analytical performance of the miRNA biosensor were characterized with stripping square-wave voltammetry (SSWV). We also demonstrated the multiplexed detection of miRNA-21 and miRNA-141 in a spiked serum sample.

\section{EXPERIMENTAL SECTION}

Materials. Sulfuric Acid $\left(\mathrm{H}_{2} \mathrm{SO}_{4}\right)$ nitric acid $\left(\mathrm{HNO}_{3}\right)$, sodium citrate, $\mathrm{NaOH}, \mathrm{NaCl}_{2}$, and neutravidin were obtained from SigmaAldrich (St. Louis, MO, USA). Autoclaved ultrapure (18.2 M $\Omega$ ) water from a Millipore Milli-Q water purification system (Billerica, MA) was used to prepare all solutions. Citrate-capped gold nanoparticles (AuNPs) were synthesized using the same protocol as previously described in the literature by citrate reduction of $\mathrm{HAuCl}_{4}{ }^{3}{ }^{1}$ Citrate-capped silver nanoparticles (AgNPs) were purchased from NanoComposix (San Diego, CA, USA)

The DNA/LNA MBs sequences were available from previous studies. $^{32,33}$ The MB consisted of a $3^{\prime}$ end thiol group for immobilization onto the metal-NPs and the presence of the biotin at the $5^{\prime}$ end, which interacts with the immobilized neutravidin on the transducer surface forming the biotin-MB-AuNP/miRNA complex upon the presence of analyte

In order to facilitate the handling of the sample, the RNA sequences were selected according to miRBase (http://www.mirbase. org) and synthesized by biomers.net (Ulm, Germany). LNA probes were purchased from Exiqon (Vedbaek, Denmark).

MB1: 5'/5BioTEG/GGCCGTCAACATCAGTCTGATAAGCT ACGGCCTTTTTTTTTT/3ThioMC3-D/-3' (LNA bases are marked in bold)

MB2: 5'/5BioTEG/AACCCACCATCTTTACCAGACAGTGTTATTACTAGTGGGTTTTTTTTTTTT/3ThioMC3-D/-3'

miRNA-21: 5'-UAGCUUAUCAGACUGAUGUUGA-3' miRNA-141: 5'-UAACACUGUCUGGUAAAGAUGG-3' miRNA-205: 5'-UCCUUCAUUCCACCGGAGUCUGU-3' miRNA-221: 5'-AGCUACAUUGUCUGCUGGGUUUC-3'

Oligonucleotide stock solutions $(100 \mu \mathrm{M})$ were prepared using autoclaved Milli-Q water and then divided into aliquots and stored at $-20{ }^{\circ} \mathrm{C}$.

Instrumentation. SSWV measurements were carried out using an IviumStat instrument (Ivium, The Netherlands). A platinum wire and a $\mathrm{Ag} / \mathrm{AgCl} \mathrm{KCl} 3 \mathrm{M}$ (CHI Instruments) electrode were used as counter and the reference electrodes, respectively. A glassy carbon electrode (GCE) with diameter of $2 \mathrm{~mm}$ were purchased from $\mathrm{CHI}$ Instruments (Bee Cave, TX, USA). Five $\mathrm{mV}$ was applied as the amplitude of the sine wave potential.

The zeta potentials and the size of the AuNPs, AgNPs, biotin-MB1AuNP conjugates, and biotin-MB2-AgNP conjugates were measured using a Zetasizer Nano ZS90 instrument (Malvern Instruments Ltd., Worcestershire, UK) based on dynamic light scattering and were 
Scheme 1. (A) Assay Design and (B) Detection Principle of the Electrochemical Biosensor for the Multiplexed Detection of miRNAs Based on the Use of Electrochemically Encoded Responsive Nanolabels

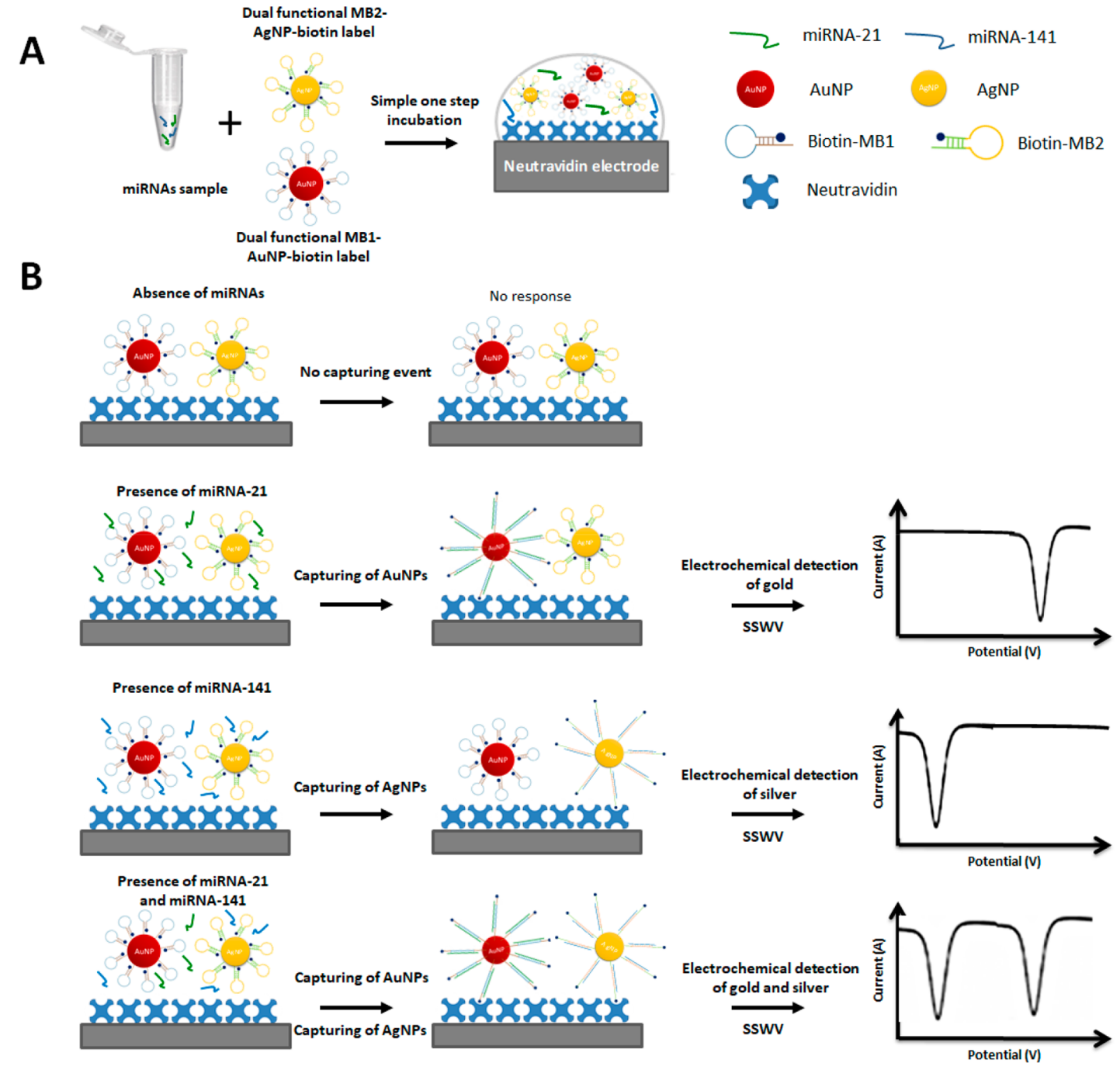

performed at room temperature $\left(20^{\circ} \mathrm{C}\right)$. Mean diameters and zeta potentials were calculated from three repeated measurements.

Scanning electron microscopy (SEM) images were recorded using a LEO 155 Gemini instrument (Zeiss, Germany).

Preparation of Biotin-MB1-AuNPs and Biotin-MB2-AgNPs Nanolabels. The biotin-MB1-AuNP and biotin-MB2-AgNP conjugates were synthesized according to a previously reported protocol. ${ }^{36}$ In brief, $250 \mu \mathrm{L}$ of the AuNPs (O.D. 2.3) or AgNPs (O.D. 2.3) dispersed in PBS (0.1 mM, pH 7.4) was mixed with an adequate volume of the biotin-MB1 and biotin-MB2 stock solution, in order to obtain a final DNA-to-NPs ratio of 500:1. The solution was then left to react at room temperature, under gentle mixing overnight. The biotin-MB1-AuNP and biotin-MB2-AgNP mixtures were subjected to an aging process that consisted of a stepwise rise of $\mathrm{NaCl}$ concentration until reaching $0.3 \mathrm{M}$. Subsequently, the obtained solutions were incubated overnight and under gentle shaking at room temperature. Then the biotin-MB1-AuNP and biotin-MB2-AgNP conjugates were washed twice by centrifugation $(11,900 \mathrm{~g}$ for $20 \mathrm{~min}$ at $20{ }^{\circ} \mathrm{C}$ ), and finally resuspend in a solution composed of PBS $(0.1$ $\mathrm{mM}, \mathrm{pH} 7.0)$ and $\mathrm{NaCl}(0.3 \mathrm{M})$ and then stored at $4{ }^{\circ} \mathrm{C}$.

Preparation of Neutravidin Electrode. Glassy carbon electrode was polished with alumina $(0.3$ and $0.05 \mu \mathrm{m})$ followed by ultrasonic cleaning in Milli- $\mathrm{Q}$ water and ethanol and drying with nitrogen gas. The neutravidin electrode was prepared by applying a $5 \mu \mathrm{L}$ of neutravidin solution $(0.25 \mathrm{mg} / \mathrm{mL})$ via the drop-cast technique, and then kept to dry at room temperature for $1 \mathrm{~h}^{24}$ Moreover, The dried neutravidin electrode was cross-linked with glutaraldehyde vapor in a sealed container for $45 \mathrm{~min}$. Finally, the prepared neutravidin electrodes were washed with PBS and dried with nitrogen gas. Before experiment, the neutravidin electrodes were rehydrated with PBS for 5 min prior to use.

Multiplex Detection of miRNA-21 and miRNA-141. Multiplexed detection of miRNAs was performed by incubating the neutravidin electrodes in a solution mixture composed of miRNA-21 and miRNA-141 together with the biotin- MB1-AuNPs and biotinMB2-AgNP nanolabels. The solution was prepared by mixing the desired amounts of target miRNAs with optimized volumes of biotinMB1-AuNPs and biotin-MB2-AgNPs in $10 \mathrm{mM} \mathrm{PB} \mathrm{(pH} \mathrm{7.5)}$ supplemented with $500 \mathrm{mM} \mathrm{NaCl}$ to a total volume of $25 \mu \mathrm{L}$. After the incubation step, the working electrode was rinsed with $25 \mu \mathrm{L}$ of PBS (10 mM, pH 7.4) and performed SSWV measurements. The pretreatment oxidation of AuNPs and AgNPs was conducted at $+1 \mathrm{~V}$ vs $\mathrm{Ag} / \mathrm{AgCl}$ for $180 \mathrm{~s}$ in the optimized electrolyte composed of 2:1 v/ $\mathrm{v}$ of a mixture of sulfuric acid $(0.1 \mathrm{M})$ and nitric acid $(0.1 \mathrm{M})$. Immediately after the electrochemical oxidation step, SSWV was performed with the potential scanned from +1.0 to $0 \mathrm{~V}$ (step potential $=10 \mathrm{mV}$ and frequency $=20 \mathrm{~Hz}$ ), resulting a SSWV signals generated from the reduction of silver ions and gold ions at $+0.33 \mathrm{~V}$ and $+0.73 \mathrm{~V}$ that corresponded to the presence of miRNA-21 and mi-RNA-141, respectively. 
Table 1. Comparison of Different Methods for Multiplex Detection of miRNAs

\begin{tabular}{|c|c|c|c|c|c|c|}
\hline $\begin{array}{l}\text { biorecognition } \\
\text { molecules immobilized } \\
\text { on electrode }\end{array}$ & $\begin{array}{l}\text { electrode for } \\
\text { detection of other } \\
\text { miRNAs }\end{array}$ & label & amplification or addition steps & target miRNAs & linear range ${ }^{a}$ & ref \\
\hline $\begin{array}{l}\text { single generic } \\
\text { neutravidin probe }\end{array}$ & yes, generic & $\begin{array}{l}\text { biotin-MB-metal } \\
\text { NPs }\end{array}$ & no, single one-pot assay approach & miR-141: LoD $10 \mathrm{pM}$ & miR-141: $50 \mathrm{pM}$ to $1 \mathrm{nM}$ & $\begin{array}{l}\text { this } \\
\text { work }\end{array}$ \\
\hline $\begin{array}{l}\text { multiple miRNA } \\
\text { capture probes }\end{array}$ & $\begin{array}{l}\text { no, required } \\
\text { different probes }\end{array}$ & doped silica NPs & yes $^{b}$ & $\begin{array}{l}\text { miR-21: LoD } 0.3 \mathrm{pM} \\
\text { miR-141: LoD } 6.3 \mathrm{fM}\end{array}$ & $\begin{array}{l}\operatorname{miR}-21: 0.5 \mathrm{pM} \text { to } 1 \mathrm{nM} \\
\text { miR-141: } 0.02-150 \mathrm{pM}\end{array}$ & 25 \\
\hline \multirow[t]{2}{*}{$\begin{array}{l}\text { multiple miRNA } \\
\text { capture probes }\end{array}$} & $\begin{array}{l}\text { no, required } \\
\text { different probes }\end{array}$ & $\begin{array}{l}\text { oligonucleotide } \\
\text { coupled liposomes }\end{array}$ & yes $^{c}$ & $\begin{array}{l}\text { miR-21: LoD } 8.6 \mathrm{fM} \\
\text { miR-221: NA }\end{array}$ & $\begin{array}{l}\operatorname{miR}-21: 0.03-150 \mathrm{pM} \\
\text { miR-221: } 0.1 \mathrm{fM} \text { to } 1 \mathrm{pM}\end{array}$ & 26 \\
\hline & & & & miR-124a: NA & miR-124a: $0.5 \mathrm{fM}$ to $1 \mathrm{pM}$ & \\
\hline $\begin{array}{l}\text { multiple miRNA } \\
\text { capture probes }\end{array}$ & $\begin{array}{l}\text { no, required } \\
\text { different probes }\end{array}$ & $\begin{array}{l}\text { oligonucleotide } \\
\text { coupled magnetic } \\
\text { NPs }\end{array}$ & $\begin{array}{l}\text { yes, hybridization chain reactions } \\
\text { (required multiple steps) }\end{array}$ & $\begin{array}{l}\text { miR-21: LoD } 0.1 \mathrm{fM} \\
\text { miR-141: LoD } 0.28 \mathrm{fM}\end{array}$ & $\begin{array}{l}\operatorname{miR}-21: 0.1 \mathrm{fM} \text { to } 1 \mathrm{pM} \\
\text { miR-141: } 1 \mathrm{fM} \text { to } 1 \mathrm{pM}\end{array}$ & 28 \\
\hline \multirow[t]{2}{*}{$\begin{array}{r}\text { multiple miRNA } \\
\text { capture probes }\end{array}$} & $\begin{array}{l}\text { no, required } \\
\text { different probes }\end{array}$ & $\begin{array}{l}\text { oligonucleotide } \\
\text { coupled magnetic/ } \\
\text { QD NPs }\end{array}$ & $\begin{array}{l}\text { yes, ligase chain reactions (required } \\
\text { heating at } 90^{\circ} \mathrm{C} \text { ) }\end{array}$ & $\begin{array}{l}\text { miR-27b: LoD } 0.36 \mathrm{fM} \\
\text { miR-155: LoD } 12 \mathrm{fM}\end{array}$ & $\begin{array}{l}\text { miR-27b: } 1 \mathrm{fM} \text { to } 1 \mathrm{pM} \\
\text { miR-155: } 50 \mathrm{fM} \text { to } 30 \mathrm{pM}\end{array}$ & 27 \\
\hline & & & & miR-27b: LoD $31 \mathrm{fM}$ & miR-27b: $50 \mathrm{fM}$ to $1 \mathrm{nM}$ & \\
\hline \multirow[t]{2}{*}{$\begin{array}{r}\text { multiple miRNA } \\
\text { capture probes }\end{array}$} & $\begin{array}{l}\text { no, required } \\
\text { different probes }\end{array}$ & $\begin{array}{l}\text { oligonucleotide } \\
\text { coupled redox } \\
\text { labels }\end{array}$ & $\begin{array}{l}\text { yes, duplex specific nuclease- } \\
\text { assisted amplifications (required } \\
\text { multiple step) }\end{array}$ & miR-141: LoD $4.2 \mathrm{fM}$ & $\mathrm{miR}-141: 5 \mathrm{fM}$ to $50 \mathrm{pM}$ & 29 \\
\hline & & & & miR-21: LoD $3.0 \mathrm{fM}$ & miR-21: $5 \mathrm{fM}$ to $50 \mathrm{pM}$ & \\
\hline
\end{tabular}

\section{Table 2. Average Hydrodynamic Diameters and Zeta Potential Measurements}

$\begin{array}{lrcrrr}\text { structure } & \text { citrate-AuNPs } & \text { biotin-MB1-AuNPs } & \begin{array}{c}\text { biotin-MB1AuNP/ } \\ \text { miRNA-21 }\end{array} & \begin{array}{c}\text { citrate-AgNPs } \\ \text { biotin-MB2-AgNPs }\end{array} & \begin{array}{c}\text { biotin-MB2-AgNP/ } \\ \text { miRNA-141 }\end{array} \\ \text { size }(\mathrm{nm}) & 29.3 \pm 0.6 & 40.3 \pm 1.9 & 55.3 \pm 2.4 & 24.1 \pm 2.3 & 36.7 \pm 1.5 \\ \text { zeta potential }(\mathrm{mV}) & -27.3 \pm 2.5 & -35.3 \pm 1.4 & -42.3 \pm 2.6 & -32.3 \pm 1.2 & -39.5 \pm 1.7\end{array}$

\section{RESULTS AND DISCUSSION}

Principle of the Neutravidin Biosensor for Simultaneous Multiplexed Detection of miRNAs. Scheme 1 illustrates the working principle of the developed neutravidin biosensor for multiplexed detection of miRNAs. It is based on a simple one-pot assay approach where simultaneous detection of miRNAs occurred upon the addition of a sample mixture of miRNA-21/biotin-MB1-AuNP and miRNA-141/biotin-MB2AgNP onto the neutravidin electrode followed by SSWV. In the absence of miRNA-21 and miRNA-141, the biotin-MB1 and biotin-MB2 retained their stem-loop structure where the biotin groups are sterically hindered and avoid interacting with the neutravidin molecules on the electrode surface. In contrast, the presence of target miRNAs, hybridization take place between the miRNA and biotin-MB causing the opening of the MB loops making the biotin terminals accessible. The activated biotin-MB1-AuNPs/miRNA-21 and biotin-MB2-AgNPs/ miRNA-141 complexes were then captured via biotinneutravidin interaction by the neutravidin-modified electrode and measured by SSWV. The binding of the metal NPs labels results in the increase of the different peak currents corresponding to AuNPs and AgNPs. This allowed different signal responses from respective miRNAs to be differentiated due the intrinsic electrochemical signature of the nanolabels. The neutravidin electrode approach provides a facile and stable biosensor platform compared with the conventional multiple oligonucleotide probe-based biosensors for multiplex miRNA detection (Table 1). Moreover, the multiplexing capacity of the neutravidin biosensor is not limited by the complicity of the immobilization chemistry compared with the multiple oligonucleotide probes approach. This generic neutravidin biosensor coupled with responsive metal-NP labels could potentially be adopted for the detection of different combinations of miRNAs by simply using different sequences of MBs on the metal-NP labels. Moreover, the signal generation mechanism is driven by a strong biotin-neutravidin interaction to give a stable signal (i.e., once the biotin-MBmetal-NP is opened and captured by neutravidin electrode surface, the biotin-neutravidin interaction dominated, and becomes independent of the relatively unstable miRNA/MB hybridization).

Characterization of the Biotin-MB1-AuNP and BiotinMB2-AgNP Labels, and Neutravidin Electrode. The immobilization of MB onto the metal NP labels and the MB opening mechanism response to miRNAs were characterized by measuring the changes is the size of the synthesized citratecapped AuNPs, biotin-MB1-AuNP label, and biotin-MB1AuNP/miRNA-21 complexes, as well as the citrate-capped AgNPs, biotin-MB2-AgNP label, and biotin-MB2-AgNP/ miRNA-141 complexes, respectively. Moreover, the surface charge densities of the corresponding NPs were characterized using zeta potential measurements. Table 2 summarizes the average hydrodynamic diameters and zeta potentials obtained.

Hydrodynamic diameters for the biotin-MB1-AuNPs (40.3 $\pm 1.9 \mathrm{~nm})$ and biotin-MB2-AgNPs $(36.7 \pm 1.5 \mathrm{~nm})$ were larger compared with the AuNPs $(29.3 \pm 0.6 \mathrm{~nm})$ and AgNPs 
$(24.1 \pm 2.3 \mathrm{~nm})$, respectively. The increase in diameter indicated the successful immobilization of MB1 and MB2 onto the AuNPs and AgNPs, respectively. Upon the addition of miRNAs sample, the hydrodynamic diameter of the biotinMB1-AuNP/miRNA-21 complexes $(55.3 \pm 2.4 \mathrm{~nm})$ and the biotin-MB2-AgNP/miRNA-141 complexes $(47.1 \pm 2.2 \mathrm{~nm})$ further increased, demonstrated the opening of the MBs stemloop structure.

Following functionalization of the AuNPs with MB1 and AgNPs with MB2, the zeta potential of the bare AuNPs increased from $-27.3 \pm 2.5$ to $-35.3 \pm 1.4 \mathrm{mV}$ (biotin-MBAuNPs) and from $-32.3 \pm 1.2$ to $-39.5 \pm 1.7 \mathrm{mV}$ (biotinMB-AgNPs), respectively. After hybridization with target miRNAs, the zeta potential of the biotin-MB1-AuNPs/ miRNA-2 1 and biotin-MB2-AgNP/miRNA-141 complexes further increased to $-42.3 \pm 2.6 \mathrm{mV}$ and $-45.3 \pm 1.8 \mathrm{mV}$, respectively. The increase in zeta potential is likely due to the negative charge properties of the nucleic acid backbone of MBs and miRNAs. The surface morphology of the neutravidin modified electrode was characterized by SEM. Figure S1 shows the SEM images of (A) a bare electrode and (B) a neutravidin modified electrode. The surface morphology of the bare electrode appeared smooth and clean, while the neutravidin modified electrode consisted of a layer of clustered structures resulting from the immobilized neutravidin molecules.

Optimization of the Voltammetric Biosensor for Multiplexed Detection of miRNAs. In order to improve the performance of the proposed biosensing approach for multiplexed detection of miRNAs, optimization of (i) the sulfuric acid $(0.1 \mathrm{M}) /$ nitric acid $(0.1 \mathrm{M})$ ratio, (ii) the concentration of biotin-MB1-AuNP and biotin-MB2-AgNP nanolabels, (iii) the pretreatment time for electrochemical oxidation of AuNPs and AgNPs into Au and Ag ions, and (iv) the pretreatment potential was performed.

To optimize the composition of the supporting electrolyte, optimization of the sulfuric acid $(0.1 \mathrm{M}) /$ nitric acid $(0.1 \mathrm{M})$ ratio was achieved by preparing electrolytes with various ratios $(1: 1,2: 1,3: 1 \mathrm{v} / \mathrm{v})$. The responses of the sensors to $0.5 \times 10^{-9}$ $\mathrm{M}$ miRNA-21 and $0.5 \times 10^{-9} \mathrm{M}$ miRNA-141 are presented in Figure 1A. The normalized signal for the detection of targeted miRNAs increased using 2:1 v/v mixture of sulfuric acid and nitric acid. However, one can observe a significant decrease in the signal response of the sensor to $0.5 \times 10^{-9} \mathrm{M}$ miRNA-141 using a ratio of $3: 1 \mathrm{v} / \mathrm{v}$ to $0.15 \mathrm{mg} / \mathrm{mL}$, while there is not a significant improvement on the signal response for miRNA- 21 . Therefore, a ratio of $2: 1 \mathrm{v} / \mathrm{v}$ of a mixture of sulfuric acid $(0.1$ $\mathrm{M})$ and nitric acid $(0.1 \mathrm{M})$ was used as the supporting electrolyte.

The amount of biotin-MB1-AuNP and biotin-MB2-AgNP nanolabels was optimized in the presence of miRNA-21 and miRNA-141 using different volumes $(4-7 \mu \mathrm{L})$ of nanolabels (OD 2.3). The normalized signal for the detection of miRNA$21\left(0.5 \times 10^{-9} \mathrm{M}\right)$ and miRNA-141 $\left(0.5 \times 10^{-9} \mathrm{M}\right)$ increased with increasing the amount of the nanolabels. The peak currents remain relatively stable after using $7 \mu \mathrm{L}$ of the nanolabels. This may be due a saturation effect on the signal response in the present of high concentration of nanolabels (Figure 1B). Thus, higher normalized signals and, subsequently, better discrimination were recorded for the $6 \mu \mathrm{L}$ of biotin-MB1-AuNP and $6 \mu \mathrm{L}$ of biotin-MB2-AgNP.

The influence of pretreatment time and pretreatment potential for the SSWV detection of miRNA-21 and miRNA141 were studied. The effect on the pretreatment time was
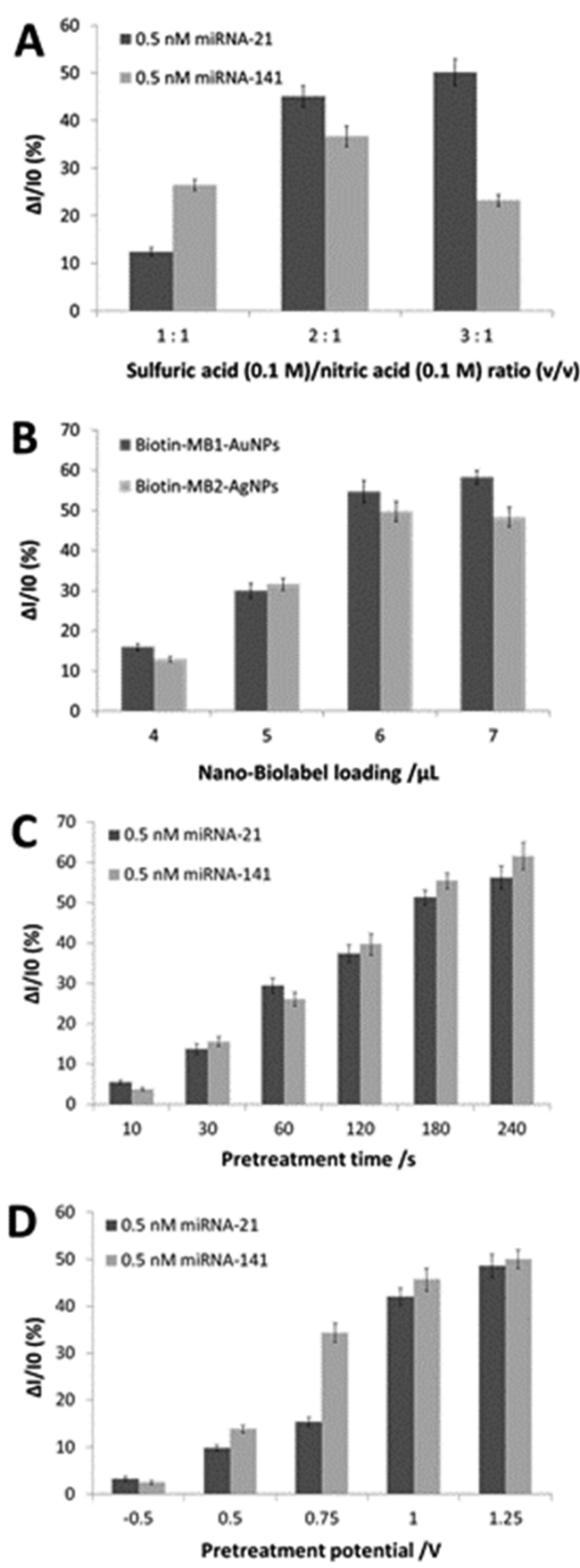

Figure 1. Optimization of the miRNA biosensor: (A) sulfuric acid/ nitric acid ratio ( $E=1 \mathrm{~V}, t=180 \mathrm{~s}, f=20 \mathrm{~Hz}$, and $n=3)$; (B) amount of nanolabels ( $E=1 \mathrm{~V}, t=180 \mathrm{~s}, f=20 \mathrm{~Hz}$, and $n=3)$; (C) pretreatment time for stripping voltammetry $(E=1 \mathrm{~V}, f=20 \mathrm{~Hz}$, and $n=3)$; and (D) pretreatment potential ( $t=180 \mathrm{~s}, f=20 \mathrm{~Hz}$, and $n=$ 3).

optimized with various time points of $10,30,60,120,180$, and $240 \mathrm{~s}$ at a fixed frequency and potential $(20 \mathrm{~Hz}$ and $1 \mathrm{~V})$. When the pretreatment time increases, the corresponding signal response increased up to 180 s (Figure 1C). While there was no significant increase in signal response when the pretreatment time was further increased to $240 \mathrm{~s}$. Therefore, $180 \mathrm{~s}$ was chosen as the optimized pretreatment time.

The pretreatment potential was optimized within a potential window of -0.5 to $1.25 \mathrm{~V}$ at fixed frequency $(20 \mathrm{~Hz})$ and pretreatment time $(180 \mathrm{~s})$. Figure 1D shows that the peak 
currents for the detection of $0.5 \times 10^{-9} \mathrm{M}$ miRNA-21 and 0.5 $\times 10^{-9} \mathrm{M}$ miRNA-141 increased as the pretreatment potential increased, reaching a maximum signal at a pretreatment potential of $1.25 \mathrm{~V}$. However, there is no significant increase in signal when changing the pretreatment potential between 1 and $1.25 \mathrm{~V}$. Therefore, a pretreatment potential of $1 \mathrm{~V}$ was chosen to reduce the effect of higher potentials which may damage the neutravidin electrode. The final optimized SSWV condition was set at pretreatment potential of $1 \mathrm{~V}$ and pretreatment time of $180 \mathrm{~s}$.

Analytical Performance of the Neutravidin Biosensor for Detection of miRNAs. The analytical performance of the neutravidin biosensor for detection of miRNAs was studied under the optimal experimental conditions as discussed above. In order to validate the approach, miRNA-21 and miRNA-141 were detected individually. A good response was obtained over the range $0.5-1000 \mathrm{pM}$ in the presence of miRNA-21 (Figure $2 \mathrm{~A}$ ), and over the range $50-1000 \mathrm{pM}$ in the presence of miRNA-141 (Figure 2B). Subsequently, the multiplexed detection of miRNA-21 and miRNA-141 was evaluated. Figure $2 \mathrm{C}$ illustrates the voltammograms obtained corresponding to the increasing of equal target miRNAs concentrations from 0.5 to $1000 \mathrm{pM}$. The neutravidin electrode displays a well-defined simultaneous response at +0.33 and $+0.73 \mathrm{~V}$ vs $\mathrm{Ag} / \mathrm{AgCl}$ to silver ions and gold ions, respectively.

The SSWV peak currents increased significantly with the simultaneous increase of concentrations of target miRNA-21 and miRNA-141. We obtained a linear relationship between the targeted miRNAs concentrations as a function of peak current (Figure 2D). A linear dynamic range was achieved for miRNA-2 1 from 0.5 to $1000 \mathrm{pM}$ with a coefficient of correlation $\left(R^{2}\right)$ of 0.9959 and a limit of detection (LOD) of $0.3 \mathrm{pM}(3 \sigma /$ slope; $n=3)$ (Figure $2 \mathrm{D})$. In the presence of miRNA-141, a linear dynamic range was obtained from 50$1000 \mathrm{pM}$ with $R^{2}$ of 0.9899 and a limit of detection of $10 \mathrm{pM}$ (Figure 2D). The sensitivity of the biosensor was calculated to be 4.5 and $3.7 \mathrm{nA} / \mathrm{pM}$ toward the detection of miRNA-21 and miRNA-141, respectively. The difference in the calculated LOD for miRNA-21 and miRNA-141 could be explained by the different metal nanoparticles used in the two systems (i.e., AuNPs for miRNA-21 and AgNPs for miRNA-141), which will affect the signal responses, sensitivity, and calculated LOD. If we further analyze the percentage error for each of the data points (error bar verse signal in percentage), for miRNA-21 the percentage error ranges from the lowest $6.2 \%$ (for $500 \mathrm{pM}$ ) to the highest $10.02 \%$ (for $750 \mathrm{pM}$ ), while for miRNA-141 the percentage error ranges from the lowest $5.2 \%$ (for $150 \mathrm{pM}$ ) to the highest $12.1 \%$ (for $500 \mathrm{pM}$ ). Therefore, the percentage errors for all data points are within a relatively close range $(\sim 5$ to $12 \%)$. For commercial biosensors in real industrial practice, an interassay error below $15 \%$ is considered to be good. A comparison between the developed generic neutravidin biosensor based on electrochemically encoded responsive nanolabels for multiplexed and simultaneous detection of miRNAs and earlier reported electrochemical biosensors can be found in Table 1. It is important to note that the analytical performance of our neutravidin biosensor coupled with a metal-nanoparticle detection system could potentially be enhanced with addition amplifications step reported in Table 1 such as ligase chain reactions ${ }^{27}$ and hybridization chain reactions. $^{28}$

The selectivity of the neutravidin biosensor combing the biotin-MBs-metal-NPs for the detection of target miRNA-21
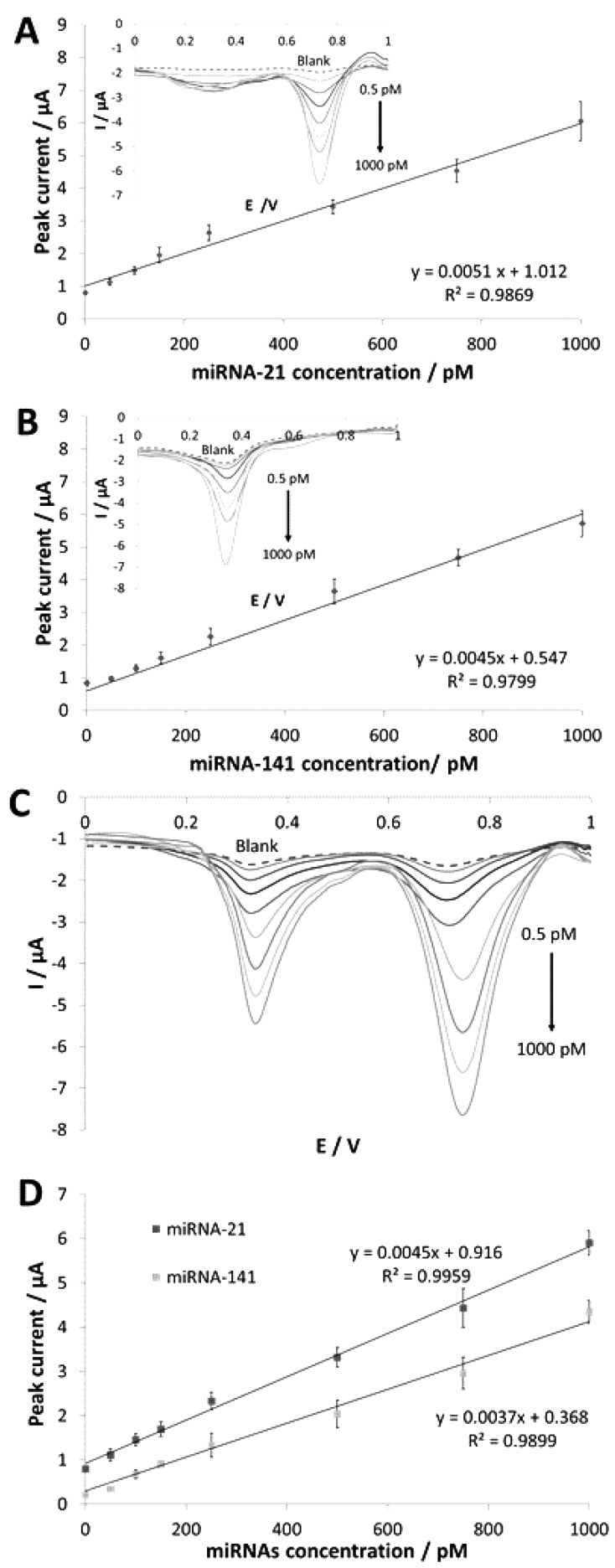

Figure 2. (A) Calibration curve of miRNA-21 ( $E=1 \mathrm{~V}, t=180 \mathrm{~s}, f=$ $20 \mathrm{~Hz}$, and $n=3$ ); inset shows obtained voltammograms for individual detection of miRNA-21. (B) Calibration curve of miRNA$141(E=1 \mathrm{~V}, t=180 \mathrm{~s}, f=20 \mathrm{~Hz}$, and $n=3)$; inset shows obtained voltammograms for individual detection of miRNA-141. (C) Voltammogram for multiplex detection of miRNA-21 and miRNA141. (D) Calibration curves for simultaneous multiplex detection of miRNA-2 1 and imRNA-141 ( $E=1 \mathrm{~V}, t=180 \mathrm{~s}, f=20 \mathrm{~Hz}$, and $n=3)$.

and miRNA-141 was studied by comparing the signal response with closely related miRNA family, miRNA-205 and miRNA211 (similarity ranging between $41 \%$ and $18 \%$ ). miRNA-205 is reported to be associated with breast, ${ }^{34}$ prostate, ${ }^{35}$ lung, ${ }^{36}$ and bladder cancer; ${ }^{37}$ while miRNA-221 is associated with bladder 
and astrocytic cancers. ${ }^{38,39}$ The selectivity study was evaluated individually in the presence of the targeted miRNA-21 and miRNA-141, respectively (Figure $3 \mathrm{~A}$ and B). A significant

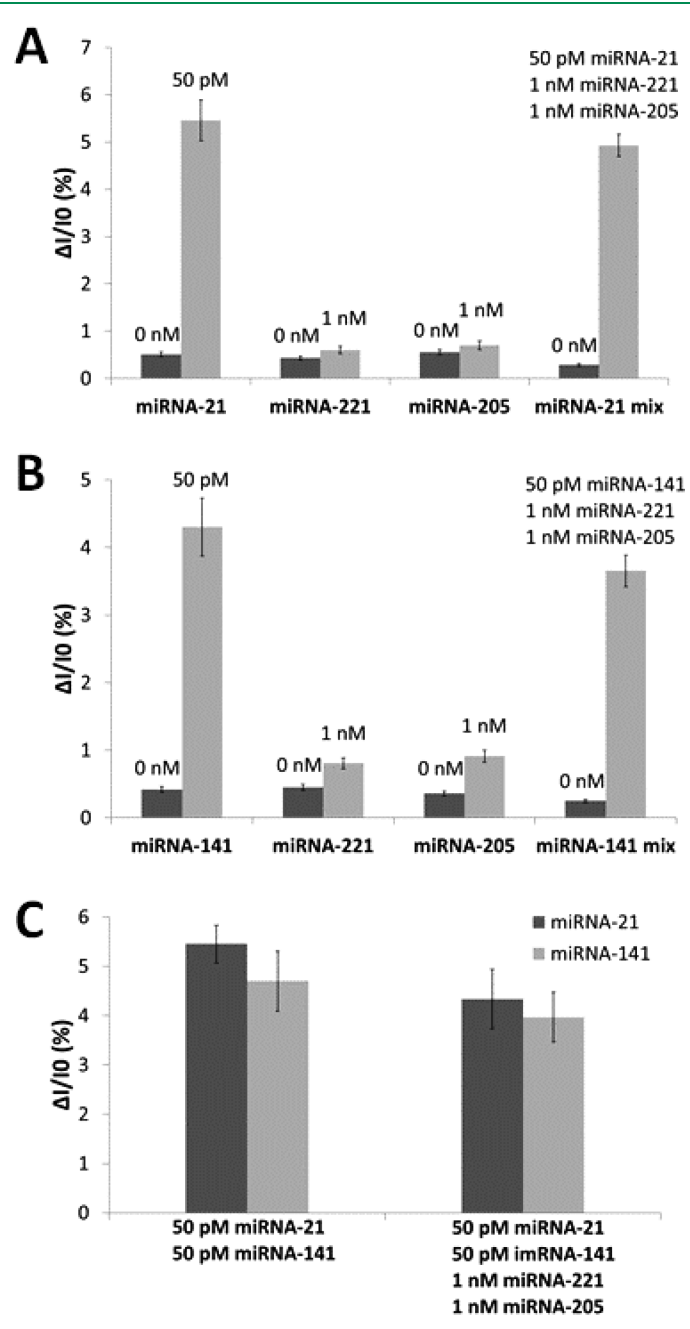

Figure 3. Selectivity study in the presence of nontargeted miRNAs (miRNA-205 and miRNA-221) toward the detection of (A) miRNA21 and (B) miRNA-141. (C) Selectivity study in the presence of nontargeted miRNAs (miRNA-205 and miRNA-221) toward the detection of equal concentration (50 pM) of miRNA-21 and miRNA$141(E=1 \mathrm{~V}, t=180 \mathrm{~s}, f=20 \mathrm{~Hz}$, and $n=3)$.

response was observed in the presence of target miRNA-21 (50 $\mathrm{pM}$ ) or miRNA-141 (50 pM), while no significant signal was observed in the presence of nontarget miRNA-205 and miRNA-221 (note: the nonspecific miRNAs were presented at a significantly higher concentration $(1 \mathrm{nM}))$. This result demonstrated a good specificity of the MBs in the biotin-MB1AuNP and biotin-MB2-AgNP labels toward the detection of miRNA-21 and miRNA-141, respectively. Moreover, Figure $3 \mathrm{C}$ shows a clear signal response obtained from of a mixture of target miRNA-21 and miRNA-141 (50 pM) and the corresponding signal response in the presence of high concentration of nonspecific miRNAs (1 nM miRNA-221 and $1 \mathrm{nM}$ miRNA-205). The selectivity study was designed taking into consideration that miRNAs are specific biomarkers in body fluids, where the diagnostic information is quite different from conventional genetic analysis because singlebased mismatch is not relevant for miRNAs.
Detection of miRNA-21 and miRNA-141 in Spiked Serum Sample. We further demonstrated the multiplexed detection of miRNA-21 and miRNA-141 in spiked serum samples with a standard addition method. Serum samples were spiked with equal concentrations of miRNA-21 and miRNA141 (50, 250, and $500 \mathrm{pM})$. Serum samples with a spiked concentration of 50, 250, and 500 pM miRNA-21 and miRNA141 were mixed with the biotin-MB1-AuNPs and biotin-MB2AgNPs and applied to the neutravidin biosensors. The signal responses from the spiked samples were recorded, and the measured miRNA concentrations were calculated by fitting to the calculated curves.

Table 3 summarizes the measured miRNA concentrations and the actual miRNA concentrations in the spiked serum

Table 3. Actual and Measured Concentrations of miRNA-21 and miRNA-141 in Spiked Serum Samples

\begin{tabular}{cccc} 
& actual concn & measured concn & RSD (\%) \\
miRNA-21 & $50 \mathrm{pM}$ & $57.2 \pm 5.3$ & 9.3 \\
& $250 \mathrm{pM}$ & $261.4 \pm 20.8$ & 7.9 \\
miRNA-141 & $500 \mathrm{pM}$ & $512.6 \pm 43.5$ & 8.5 \\
& $50 \mathrm{pM}$ & $62.4 \pm 7.2$ & 11.5 \\
& $250 \mathrm{pM}$ & $259.8 \pm 28.4$ & 10.9 \\
& $500 \mathrm{pM}$ & $515.3 \pm 65.9$ & 12.7 \\
\hline
\end{tabular}

samples, where the actual concentration is defined as the spiked concentration of the targeted miRNAs in the serum sample, while the measured concentration is the calculated concentration obtained using the standard addition method. The correlation plots on the measured miRNA concentrations vs the actual miRNA concentrations are shown in Figure 4. There was a good match between the measured experimental values and the nominal concentration of the miRNA-21 and miRNA-141 in the serum samples. Thus, our developed
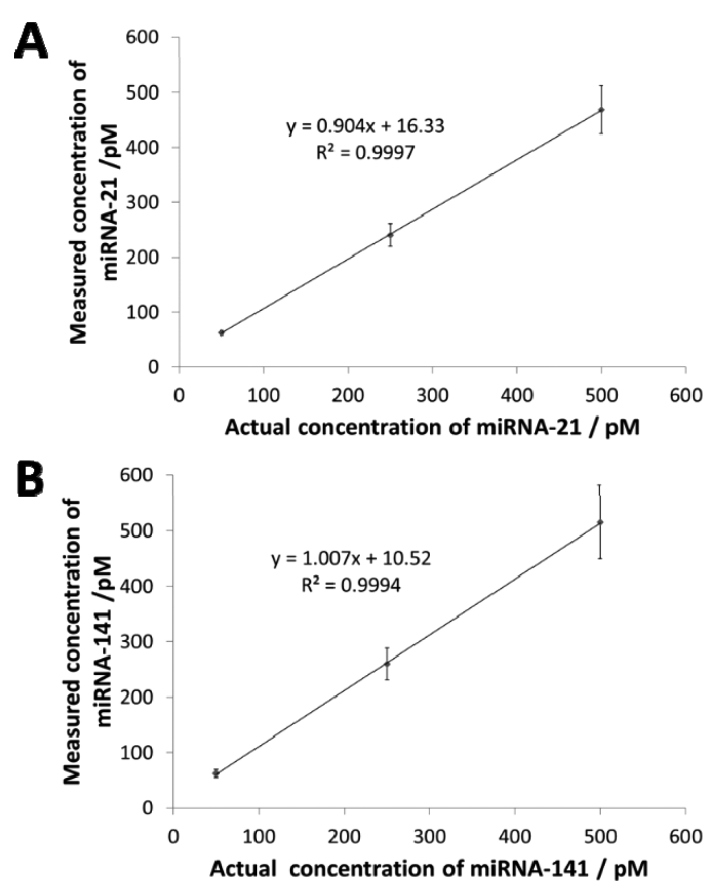

Figure 4. Correlation plot between measured and actual concentrations of (A) miRNA-21 and (B) miRNA-141 in spiked serum samples $(E=1 \mathrm{~V}, t=180 \mathrm{~s}, f=20 \mathrm{~Hz}$, and $n=3)$. 
biosensor allowed accurate multiplexed detection of miRNA21 and miRNA-141 in spiked serum samples.

\section{CONCLUSIONS}

We have demonstrated a simple generic neutravidin biosensor combined with electrochemically encoded responsive nanolabels for simultaneous multiplexed detection of miRNA-21 and miRNA-141. The electrochemically encoded responsive nanolabels consisted of AuNPs and AgNPs coupled with biotin-MB for both biorecognition and signal generation. A linear dynamic range was achieved for miRNA-21 from 0.5 to $1000 \mathrm{pM}$, and the limit of detection was calculated to be 0.3 pM; and for miRNA-141 from 50 to 1000 pM with a limit of detection of $10 \mathrm{pM}$. Moreover, we demonstrated the multiplexed detection of miRNA-21 and miRNA-141 in spiked serum. This generic and robust neutravidin biosensor potentially allows for the detection of different combinations of miRNAs by simply using different sequences of $\mathrm{MB}$ probes to differentiate the various signal response from different miRNAs via the intrinsic electrochemical signature of the nanolabels. This innovative generic biosensor design simplifies the transducer preparation for multiplexed detection of miRNAs while offering simple operation and good analytical performance. This approach could accelerate the biosensor development time for the detection of newly discovered miRNAs for various diagnostic applications.

\section{ASSOCIATED CONTENT}

\section{(5 Supporting Information}

The Supporting Information is available free of charge on the ACS Publications website at DOI: 10.1021/acssensors.8b00942.

SEM images of electrodes (PDF)

\section{AUTHOR INFORMATION}

\section{Corresponding Author}

*E-mail: wing.cheung.mak@liu.se.

\section{ORCID}

Wing Cheung Mak: 0000-0003-3274-6029

\section{Present Address}

${ }^{\perp}$ A.P.F.T.: SATM, Cranfield University, Bedfordshire, MK430AL, UK.

\section{Author Contributions}

"S.A. and Z.F.: Equal contribution with first authorship.

\section{Notes}

The authors declare no competing financial interest.

\section{ACKNOWLEDGMENTS}

We are thankful for the financial support by the EU-FP7 project PIRSES-GA-2012-318053.

\section{REFERENCES}

(1) Lee, R. C.; Feinbaum, R. L.; Ambros, V. The C. Elegans Heterochronic Gene Lin-4 Encodes Small RNAs with Antisense Complementarity to Lin-14. Cell 1993, 75 (5), 843-854.

(2) Wightman, B.; Ha, I.; Ruvkun, G. Posttranscriptional Regulation of the Heterochronic Gene Lin-14 by Lin-4 Mediates Temporal Pattern Formation in C. Elegans. Cell 1993, 75 (5), 855-862.

(3) Pasquinelli, A. E.; Reinhart, B. J.; Slack, F.; Martindale, M. Q.; Kuroda, M. I.; Maller, B.; Hayward, D. C.; Ball, E. E.; Degnan, B.; Müller, P.; et al. Conservation of the Sequence and Temporal
Expression of Let-7Heterochronic Regulatory RNA. Nature 2000, 408 (6808), 86-89.

(4) Wienholds, E.; Kloosterman, W. P.; Miska, E.; Alvarez-Saavedra, E.; Berezikov, E.; de Bruijn, E.; Horvitz, H. R.; Kauppinen, S.; Plasterk, R. H. A. MicroRNA Expression in Zebrafish Embryonic Development. Science 2005, 309 (5732), 310-311.

(5) Bartel, D. P. MicroRNAs: Genomics, Biogenesis, Mechanism, and Function. Cell 2004, 116 (2), 281-297.

(6) Filipowicz, W.; Bhattacharyya, S. N.; Sonenberg, N. Mechanisms of Post-Transcriptional Regulation by MicroRNAs: Are the Answers in Sight? Nat. Rev. Genet. 2008, 9 (2), 102-114.

(7) Liu, X.; He, S.; Skogerbø, G.; Gong, F.; Chen, R. Integrated Sequence-Structure Motifs Suffice to Identify MicroRNA Precursors. PLoS One 2012, 7 (3), No. e32797.

(8) Catuogno, S.; Esposito, C. L.; Quintavalle, C.; Cerchia, L.; Condorelli, G.; De Franciscis, V. Recent Advance in Biosensors for MicroRNAs Detection in Cancer. Cancers 2011, 3 (2), 1877-1898.

(9) Zhou, S.-S.; Jin, J.-P.; Wang, J.-Q.; Zhang, Z.-G.; Freedman, J. H.; Zheng, Y.; Cai, L. MiRNAS in Cardiovascular Diseases: Potential Biomarkers, Therapeutic Targets and Challenges. Acta Pharmacol. Sin. 2018, 39 (7), 1073-1084.

(10) Chen, J.-Q.; Papp, G.; Szodoray, P.; Zeher, M. The Role of MicroRNAs in the Pathogenesis of Autoimmune Diseases. Autoimmun. Rev. 2016, 15 (12), 1171-1180.

(11) Shah, P.; Cho, S. K.; Thulstrup, P. W.; Bjerrum, M. J.; Lee, P. H.; Kang, J.-H.; Bhang, Y.-J.; Yang, S. W. MicroRNA Biomarkers in Neurodegenerative Diseases and Emerging NanoSensors Technology. J. Mov. Disord. 2017, 10 (1), 18-28.

(12) Mitchell, P. S.; Parkin, R. K.; Kroh, E. M.; Fritz, B. R.; Wyman, S. K.; Pogosova-Agadjanyan, E. L.; Peterson, A.; Noteboom, J.; O’Briant, K. C.; Allen, A.; et al. Circulating MicroRNAs as Stable Blood-Based Markers for Cancer Detection. Proc. Natl. Acad. Sci. U. S. A. 2008, 105 (30), 10513-10518.

(13) Kim, Y.-K. Extracellular MicroRNAs as Biomarkers in Human Disease. Chonnam Med. J. 2015, 51 (2), 51-57.

(14) Chen, X.; Ba, Y.; Ma, L.; Cai, X.; Yin, Y.; Wang, K.; Guo, J.; Zhang, Y.; Chen, J.; Guo, X.; et al. Characterization of MicroRNAs in Serum: A Novel Class of Biomarkers for Diagnosis of Cancer and Other Diseases. Cell Res. 2008, 18 (10), 997-1006.

(15) Kim, S. W.; Li, Z.; Moore, P. S.; Monaghan, A. P.; Chang, Y.; Nichols, M.; John, B. A Sensitive Non-Radioactive Northern Blot Method to Detect Small RNAs. Nucleic Acids Res. 2010, 38 (7), No. e98.

(16) Chen, C.; Tan, R.; Wong, L.; Fekete, R.; Halsey, J. Quantitation of MicroRNAs by Real-Time RT-QPCR. Methods Mol. Biol. 2011, 687, 113-134.

(17) Li, W.; Ruan, K. MicroRNA Detection by Microarray. Anal. Bioanal. Chem. 2009, 394 (4), 1117-1124.

(18) Ma, D.; Huang, C.; Zheng, J.; Tang, J.; Li, J.; Yang, J.; Yang, R. Quantitative Detection of Exosomal MicroRNA Extracted from Human Blood Based on Surface-Enhanced Raman Scattering. Biosens. Bioelectron. 2018, 101, 167-173.

(19) Tanaka, K.; Tainaka, K.; Umemoto, T.; Nomura, A.; Okamoto, A. An Osmium-DNA Interstrand Complex: Application to Facile DNA Methylation Analysis. J. Am. Chem. Soc. 2007, 129 (46), 14511-14517.

(20) Bettazzi, F.; Hamid-Asl, E.; Esposito, C. L.; Quintavalle, C.; Formisano, N.; Laschi, S.; Catuogno, S.; Iaboni, M.; Marrazza, G.; Mascini, M.; et al. Electrochemical Detection of MiRNA-222 by Use of a Magnetic Bead-Based Bioassay. Anal. Bioanal. Chem. 2013, 405 (2-3), 1025-1034.

(21) Salahandish, R.; Ghaffarinejad, A.; Omidinia, E.; Zargartalebi, H.; Majidzadeh-A, K.; Naghib, S. M.; Sanati-Nezhad, A. Label-Free Ultrasensitive Detection of Breast Cancer MiRNA-21 Biomarker Employing Electrochemical Nano-Genosensor Based on Sandwiched AgNPs in PANI and N-Doped Graphene. Biosens. Bioelectron. 2018, $120,129-136$

(22) Yin, H.; Zhou, Y.; Zhang, H.; Meng, X.; Ai, S. Electrochemical Determination of MicroRNA-21 Based on Graphene, LNA Integrated 
Molecular Beacon, AuNPs and Biotin Multifunctional Bio Bar Codes and Enzymatic Assay System. Biosens. Bioelectron. 2012, 33 (1), 247253.

(23) Kangkamano, T.; Numnuam, A.; Limbut, W.; Kanatharana, P.; Vilaivan, T.; Thavarungkul, P. Pyrrolidinyl PNA Polypyrrole/Silver Nanofoam Electrode as a Novel Label-Free Electrochemical MiRNA21 Biosensor. Biosens. Bioelectron. 2018, 102, 217-225.

(24) Fredj, Z.; Azzouzi, S.; Turner, A. P. F.; Ali, M. B.; Mak, W. C. Neutravidin Biosensor for Direct Capture of Dual-Functional BiotinMolecular Beacon-AuNP Probe for Sensitive Voltammetric Detection of MicroRNA. Sens. Actuators, B 2017, 248, 77-84.

(25) Feng, X.; Gan, N.; Zhang, H.; Li, T.; Cao, Y.; Hu, F.; Jiang, Q. Ratiometric Biosensor Array for Multiplexed Detection of MicroRNAs Based on Electrochemiluminescence Coupled with Cyclic Voltammetry. Biosens. Bioelectron. 2016, 75, 308-314.

(26) Ghazizadeh, E.; Hosseinkhani, S.; Oskuee, R. K.; Molaabasi, F.; Jaafari, M. R. Sequential or Multiplex Electrochemical Detection of MiRs Based on the P19 Function Relative to Three Sandwiches of Different Structural Hybrids on the Liposomal Sensor. Mater. Sci. Eng., C 2018, 92, 703-711.

(27) Zhu, W.; Su, X.; Gao, X.; Dai, Z.; Zou, X. A Label-Free and PCR-Free Electrochemical Assay for Multiplexed MicroRNA Profiles by Ligase Chain Reaction Coupling with Quantum Dots Barcodes. Biosens. Bioelectron. 2014, 53, 414-419.

(28) Yuan, Y.-H.; Wu, Y.-D.; Chi, B.-Z.; Wen, S.-H.; Liang, R.-P.; Qiu, J.-D. Simultaneously Electrochemical Detection of MicroRNAs Based on Multifunctional Magnetic Nanoparticles Probe Coupling with Hybridization Chain Reaction. Biosens. Bioelectron. 2017, 97, 325-331.

(29) Yang, C.; Dou, B.; Shi, K.; Chai, Y.; Xiang, Y.; Yuan, R. Multiplexed and Amplified Electronic Sensor for the Detection of MicroRNAs from Cancer Cells. Anal. Chem. 2014, 86 (23), 1191311918 .

(30) Juskowiak, B. Nucleic Acid-Based Fluorescent Probes and Their Analytical Potential. Anal. Bioanal. Chem. 2011, 399 (9), 3157-3176.

(31) Ghosh, S. K.; Pal, A.; Kundu, S.; Nath, S.; Pal, T. Fluorescence Quenching of 1-Methylaminopyrene near Gold Nanoparticles: Size Regime Dependence of the Small Metallic Particles. Chem. Phys. Lett. 2004, 395 (4), 366-372.

(32) Kor, K.; Turner, A. P. F.; Zarei, K.; Atabati, M.; Beni, V.; Mak, W. C. Structurally Responsive Oligonucleotide-Based Single-Probe Lateral-Flow Test for Detection of MiRNA-21 Mimics. Anal. Bioanal. Chem. 2016, 408 (5), 1475-1485.

(33) Jou, A. F.; Lu, C.-H.; Ou, Y.-C.; Wang, S.-S.; Hsu, S.-L.; Willner, I.; Ho, J. A. Diagnosing the MiR-141 Prostate Cancer Biomarker Using Nucleic Acid-Functionalized CdSe/ZnS QDs and Telomerase. Chem. Sci. 2015, 6 (1), 659-665.

(34) Greene, S. B.; Herschkowitz, J. I.; Rosen, J. M. The Ups and Downs of MiR-205: Identifying the Roles of MiR-205 in Mammary Gland Development and Breast Cancer. RNA Biol. 2010, 7 (3), 300304.

(35) Peng, Y.; Jiang, J.; Yu, R. A Sensitive Electrochemical Biosensor for MicroRNA Detection Based on Streptavidin-Gold Nanoparticles and Enzymatic Amplification. Anal. Methods 2014, 6 (9), 2889-2893.

(36) Majid, S.; Dar, A. A.; Saini, S.; Yamamura, S.; Hirata, H.; Tanaka, Y.; Deng, G.; Dahiya, R. MicroRNA-205-Directed Transcriptional Activation of Tumor Suppressor Genes in Prostate Cancer. Cancer 2010, 116 (24), 5637-5649.

(37) Markou, A.; Tsaroucha, E. G.; Kaklamanis, L.; Fotinou, M.; Georgoulias, V.; Lianidou, E. S. Prognostic Value of Mature MicroRNA-21 and MicroRNA-205 Overexpression in Non-Small Cell Lung Cancer by Quantitative Real-Time RT-PCR. Clin. Chem. 2008, 54 (10), 1696-1704.

(38) Wiklund, E. D.; Bramsen, J. B.; Hulf, T.; Dyrskjøt, L.; Ramanathan, R.; Hansen, T. B.; Villadsen, S. B.; Gao, S.; Ostenfeld, M. S.; Borre, M.; et al. Coordinated Epigenetic Repression of the MiR-200 Family and MiR-205 in Invasive Bladder Cancer. Int. J. Cancer 2011, 128 (6), 1327-1334.
(39) Lu, Q.; Lu, C.; Zhou, G.-P.; Zhang, W.; Xiao, H.; Wang, X.-R. MicroRNA-221 Silencing Predisposed Human Bladder Cancer Cells to Undergo Apoptosis Induced by TRAIL. Urol. Oncol. 2010, 28 (6), 635-641. 